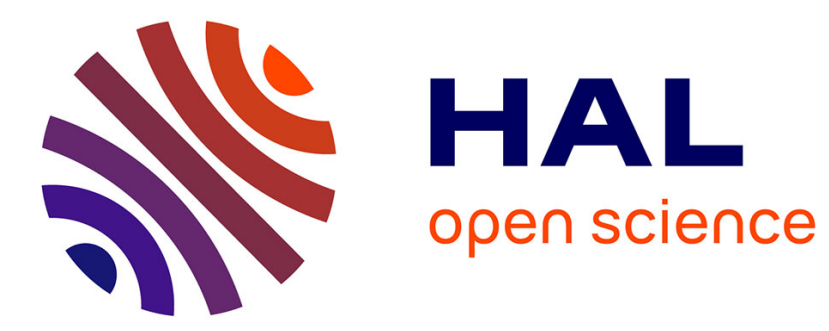

\title{
Extension of the observability rank condition to nonlinear systems driven by unknown inputs
}

\author{
Agostino Martinelli
}

\section{To cite this version:}

Agostino Martinelli. Extension of the observability rank condition to nonlinear systems driven by unknown inputs. MED 2015, Jun 2015, torremolinos, Spain. 10.1109/MED.2015.7158811 . hal01248783

\section{HAL Id: hal-01248783 \\ https://inria.hal.science/hal-01248783}

Submitted on 28 Dec 2015

HAL is a multi-disciplinary open access archive for the deposit and dissemination of scientific research documents, whether they are published or not. The documents may come from teaching and research institutions in France or abroad, or from public or private research centers.
L'archive ouverte pluridisciplinaire HAL, est destinée au dépôt et à la diffusion de documents scientifiques de niveau recherche, publiés ou non, émanant des établissements d'enseignement et de recherche français ou étrangers, des laboratoires publics ou privés. 


\title{
Extension of the Observability Rank Condition to Nonlinear systems driven by Unknown Inputs*
}

\author{
Agostino Martinelli
}

\begin{abstract}
This paper investigates the unknown input observability problem in the nonlinear case under the assumption that the unknown inputs are differentiable functions of time (up to a given order). The goal is not to design new observers but to provide simple analytic conditions in order to check the weak local observability of the state. The analysis starts by providing a new definition of indistinguishable states in the case of unknown inputs. Then, in order to separate the effect of the known inputs from the effect of the unknown inputs on the system outputs, the state is augmented. This allows us to obtain the extension of basic properties, which hold in the case of known inputs. Starting from these properties, the paper provides a sufficient analytic condition for the state observability, which is called the extended observability rank condition. The proposed approach is used to derive the observability properties of two systems. The former is very simple while the latter is very complex and describes the fusion of visual and inertial measurements.
\end{abstract}

Keywords: Nonlinear Observability; Unknown Input; Nonlinear Systems;

\section{INTRODUCTION}

The problem of state observability for systems driven by unknown inputs (UI) is a fundamental problem in control theory. This problem was introduced and firstly investigated in the seventies [3], [6], [15], [35]. A huge effort has then been devoted to design observers for both linear and nonlinear systems in presence of UI, e.g., [1], [2], [8], [9], [12], [13], [14], [17], [18], [21], [24], [25], [37].

The goal of this paper is not to design new observers for systems driven by UI but to provide simple analytic conditions in order to check the weak local observability of the state. The obtained results hold for systems whose dynamics are nonlinear in the state and affine in both the known and the unknown inputs. Additionally, the unknown inputs are supposed to be smooth functions of time (specifically, they are supposed to be $\mathcal{C}^{k}$, for a suitable integer $k$ ).

In [19] the observability properties of a nonlinear system are derived starting from the definition of indistinguishable states. According to this definition, the Lie derivatives of any output computed along any direction allowed by the system dynamics take the same values at the states which are indistinguishable. Hence, if a given state $x$ belongs to the indistinguishable set of a state $x_{0}$ (i.e., to $I_{x_{0}}$ ) all the Lie derivatives computed at $x$ and at $x_{0}$ take the same values. This is a fundamental property. In particular, based on this

\footnotetext{
*This work was supported by the French National Research Agency ANR through the project VIMAD

A. Martinelli is with INRIA Rhone Alpes, Montbonnot, France e-mail: agostino.martinelli@ieee.org
}

property, the observability rank condition was introduced in [19].

Our objective is to extend the observability rank condition to systems characterized by dynamics that are also driven by UI. For, we introduce a new definition of indistinguishable states for the case UI (section II). Then, in section III we introduce a new system by a suitable state extension. For this extended system, we show that, the Lie derivatives of the outputs up to a given order, take the same values at the states which are indistinguishable. In other words, the new system satisfies the same property derived in [19] mentioned above and this allows us to extend the observability rank condition (section IV). We will refer to this extension as to the Extended Observability Rank Condition $(E O R C)$.

The new system is obtained by a state augmentation. In particular, the augmented state is obtained by including the unknown inputs together with their time-derivatives up to given order. This augmented state has already been considered in the past. Specifically, in [4] the authors adopted this augmented state to investigate the observability properties of a fundamental problem in the framework of mobile robotics (the bearing SLAM). In particular, starting from the idea of including the time-derivatives of the unknown input in the state, in [4] a sufficient condition for the state observability has been provided.

All the theoretical results are illustrated in section $\mathrm{V}$ by deriving the observability properties of two nonlinear systems driven by unknown inputs. Finally, in section VI we provide conclusion together with some discussion with special emphasis on future research outlooks.

\section{BASIC DEFINITIONS}

In the sequel we will refer to a nonlinear control system with $m_{u}$ known inputs $\left(u \equiv\left[u_{1}, \cdots, u_{m_{u}}\right]^{T}\right)$ and $m_{w}$ unknown inputs or disturbances $\left(w \equiv\left[w_{1}, \cdots, w_{m_{w}}\right]^{T}\right)$. The state is the vector $x \in M$, with $M$ an open set of $\mathbb{R}^{n}$. We assume that the dynamics are nonlinear with respect to the state and affine with respect to the inputs (both known and unknown). Finally, for the sake of simplicity, we will refer to the case of a single output $y$ (the extension to multiple outputs is straightforward). Our system is characterized by the following equations:

$$
\left\{\begin{array}{l}
\dot{x}=f_{0}(x)+\sum_{i=1}^{m_{u}} f_{i}(x) u_{i}+\sum_{j=1}^{m_{w}} g_{j}(x) w_{j} \\
y=h(x)
\end{array}\right.
$$


where $f_{i}(x), i=0,1, \cdots, m_{u}$, and $g_{j}(x), j=1, \cdots, m_{w}$, are vector fields in $M$ and the function $h(x)$ is a scalar function defined on the open set $M$. For the sake of simplicity, we will assume that all these functions are analytic functions in $M$.

Let us consider the time interval $\mathcal{I} \equiv[0, T]$. Note that, since the equations in (1) do not depend explicitly on time, this can be considered as a general time interval of length $T$. In the sequel, we will assume that the solution of (1) exists in $\mathcal{I}$ and we will denote by $x\left(t ; x_{0} ; u ; w\right)$ the state at a given time $t \in \mathcal{I}$, when $x(0)=x_{0}$ and the known input and the disturbance are $u(t)$ and $w(t)$, respectively, $\forall t \in \mathcal{I}$.

We introduce the following definition:

Definition 1 (Indistinguishable states in presence of UI) Two states $x_{a}$ and $x_{b}$ are indistinguishable if, for any $u(t)$ (the known input vector function), there exist $w_{a}(t)$ and $w_{b}(t)$ (i.e., two unknown input vector functions in general, but not necessarily, different from each other) such that $h\left(x\left(t ; x_{a} ; u ; w_{a}\right)\right)=h\left(x\left(t ; x_{b} ; u ; w_{b}\right)\right) \forall t \in \mathcal{I}$.

This definition states that, if $x_{a}$ and $x_{b}$ are indistinguishable, then, for any known input, by looking at the output during the time interval $\mathcal{I}$, we cannot conclude if the initial state was $x_{a}$ and the disturbance $w_{a}$ or if the initial state was $x_{b}$ and the disturbance $w_{b}$. We remark that, contrary to the definition of indistinguishable states in the case without disturbances, the new definition does not establish an equivalence relation. Indeed, we can have $x_{a}$ and $x_{b}$ indistinguishable, $x_{b}$ and $x_{c}$ indistinguishable but $x_{a}$ and $x_{c}$ are not indistinguishable. As in the case of known inputs, given $x_{0}$, the indistinguishable set $I_{x_{0}}$ is the set of all the states $x$ such that $x$ and $x_{0}$ are indistinguishable. Starting from this definition, we can use exactly the same definitions of observability and weak local observability adopted in the case without disturbances.

\section{EXTENDED SYSTEM AND BASIC PROPERTIES}

In order to extend the observability rank condition to the case of unknown inputs we introduce a new system (the extended system) such that its Lie derivatives are constant on the indistinguishable sets. The new system will be denoted by $\Sigma^{(k)}$. It is simply obtained by extending the original state by including the unknown inputs together with their time derivatives. Specifically, we denote by ${ }^{k} x$ the extended state that includes the time derivatives up to the $(k-1)$-order:

$$
{ }^{k} x \equiv\left[x^{T}, w^{T}, w^{(1) T}, \cdots, w^{(k-1) T}\right]^{T}
$$

where $w^{(k)} \equiv \frac{d^{k} w}{d t^{k}}$ and ${ }^{k} x \in M^{(k)}$, with $M^{(k)}$ an open set of $\mathbb{R}^{n+k m_{w}}$. From (1) it is immediate to obtain the dynamics for the extended state:

$$
{ }^{k} \dot{x}=f_{0}^{(k)}\left({ }^{k} x\right)+\sum_{i=1}^{m_{u}} f_{i}^{(k)}(x) u_{i}+\sum_{j=1}^{m_{w}} 1_{n+k m_{w}}^{n+(k-1) m_{w}+j} w_{j}^{(k)}
$$

$$
f_{0}^{(k)}\left({ }^{k} x\right) \equiv\left[\begin{array}{c}
f_{0}(x)+\sum_{i=1}^{m_{w}} g_{i}(x) w_{i} \\
w^{(1)} \\
w^{(2)} \\
\cdots \\
w^{(k-1)} \\
0_{m_{w}} \\
f_{i}^{(k)}(x) \equiv\left[\begin{array}{c}
f_{i}(x) \\
0_{k m_{w}}
\end{array}\right]
\end{array}\right.
$$

and we denoted by $0_{m}$ the $m$-dimensional zero column vector and by $1_{m}^{l}$ the $m$-dimensional unit column vector, with 1 in the $l^{t h}$ position and 0 elsewhere. We remark that the resulting system has still $m_{u}$ known inputs and $m_{w}$ disturbances. However, while the $m_{u}$ known inputs coincide with the original ones, the $m_{w}$ unknown inputs are now the $k$-order time derivatives of the original disturbances. The state evolution depends on the known inputs via the vector fields $f_{i}^{(k)},\left(i=1, \cdots, m_{u}\right)$ and it depends on the disturbances via the unit vectors $1_{n+k m_{w}}^{n+(k-1) m_{w}+j},(j=$ $\left.1, \cdots, m_{w}\right)$. Finally, we remark that only the vector field $f_{0}^{(k)}$ depends on the new state elements.

In the rest of this section we derive several properties satisfied by $\Sigma^{(k)}$.

Lemma 1 Let us consider the system $\Sigma^{(k)}$. The Lie derivatives of the output up to the $m^{\text {th }}$ order $(m \leq k)$ are independent of $w_{j}^{(f)}, j=1, \cdots, m_{w}, \forall f \geq m$.

Proof: We proceed by induction on $m$ for any $k$. When $m=0$ we only have one zero-order Lie derivative (i.e., $h(x)$ ), which only depends on $x$, namely it is independent of $w^{(f)}, \forall f \geq 0$. Let us assume that the previous assert is true for $m$ and let us prove that it holds for $m+1$. If it is true for $m$, any Lie derivative up to the $m^{t h}$ order is independent of $w^{(f)}$, for any $f \geq m$. In other words, the analytical expression of any Lie derivative up to the $m$-order is represented by a function $g\left(x, w, w^{(1)}, \cdots, w^{(m-1)}\right)$. Hence, $\nabla g=\left[\frac{\partial g}{\partial x}, \frac{\partial g}{\partial w}, \frac{\partial g}{\partial w^{(1)}}, \cdots, \frac{\partial g}{\partial w^{(m-1)}}, 0_{(k-m) m_{w}}\right]$. It is immediate to realize that the product of this gradient by any vector filed in (3) depends at most on $w^{(m)}$, i.e., it is independent of $w^{(f)}, \forall f \geq m+1$

A simple consequence of this lemma are the following two properties:

Proposition 1 Let us consider the system $\Sigma^{(k)}$. The Lie derivatives of the output up to the $k^{\text {th }}$ order along at least one vector among $1_{n+k m_{w}}^{n+(k-1) m_{w}+j}\left(j=1, \cdots, m_{w}\right)$ are identically zero.

Proof: From the previous lemma it follows that all the Lie derivatives, up to the $(k-1)$-order are independent of $w^{(k-1)}$, which are the last $m_{w}$ components of the extended state in (2). Then, the proof follows from the fact that any vector among $1_{n+k m_{w}}^{n+(k-1) m_{w}+j}\left(j=1, \cdots, m_{w}\right)$ has the first $n+(k-1) m_{w}$ components equal to zero

where: 
Proposition 2 The Lie derivatives of the output up to the $k^{\text {th }}$ order along any vector field $f_{0}^{(k)}, f_{1}^{(k)}, \cdots, f_{m_{u}}^{(k)}$ for the system $\Sigma^{(k)}$ coincide with the same Lie derivatives for the system $\Sigma^{(k+1)}$

Proof: We proceed by induction on $m$ for any $k$. When $m=0$ we only have one zero-order Lie derivative (i.e., $h(x)$ ), which is obviously the same for the two systems, $\Sigma^{(k)}$ and $\Sigma^{(k+1)}$. Let us assume that the previous assert is true for $m$ and let us prove that it holds for $m+1 \leq k$. If it is true for $m$, any Lie derivative up to the $m^{\text {th }}$ order is the same for the two systems. Additionally, from lemma 1 , we know that these Lie derivatives are independent of $w^{(f)}, \forall f \geq m$. The proof follows from the fact that the first $n+m m_{w}$ components of $f_{0}^{(k)}, f_{1}^{(k)}, \ldots, f_{m_{u}}^{(k)}$ coincide with the first $n+m m_{w}$ components of $f_{0}^{(k+1)}, f_{1}^{(k+1)}, \cdots, f_{m_{u}}^{(k+1)}$ when $m<k$

In the sequel we will use the notation: $\xi \equiv$ $\left[w^{T}, w^{(1) T}, \cdots, w^{(k-1) T}\right]^{T}$. In this notation we have ${ }^{k} x=\left[x^{T}, \xi^{T}\right]^{T}$. We also denote by $\Sigma^{(0)}$ the original system, i.e., the one characterized by the state $x$ and the equations in (1). The definition 1 , given for $\Sigma^{(0)}$, can be applied to $\Sigma^{(k)}$. Specifically, in $\Sigma^{(k)}$, two states $\left[x_{a}, \xi_{a}\right]$ and $\left[x_{b}, \xi_{b}\right]$ are indistinguishable if, for any $u(t)$ (the known inputs), there exist two vector functions $w_{a}^{(k)}(t)$ and $w_{b}^{(k)}(t)$ (the $k^{\text {th }}$ time derivative of two disturbance vectors) such that, $h\left(x\left(t ;\left[x_{a}, \xi_{a}\right] ; u ; w_{a}^{(k)}\right)\right)=h\left(x\left(t ;\left[x_{b}, \xi_{b}\right] ; u ; w_{b}^{(k)}\right)\right)$ $\forall t \in \mathcal{I}$.

It holds the following fundamental result:

Proposition 3 If $\left[x_{a}, \xi_{a}\right]$ and $\left[x_{b}, \xi_{b}\right]$ are indistinguishable in $\Sigma^{(k)}$ then the Lie derivatives of the output up to the $k^{\text {th }}$ order computed on these points take the same values.

Proof: We consider a piecewise-constant input $\tilde{u}$ as follows $\left(i=1, \cdots, m_{u}\right)$ :

$$
\tilde{u}_{i}(t)=
$$

$\begin{cases}u_{i}^{1} & t \in\left[0, t_{1}\right) \\ u_{i}^{2} & t \in\left[t_{1}, t_{1}+t_{2}\right) \\ \cdots & \\ u_{i}^{g} & t \in\left[t_{1}+t_{2}+\cdots+t_{g-1}, t_{1}+t_{2}+\cdots+t_{g-1}+t_{g}\right)\end{cases}$

Since $\left[x_{a}, \xi_{a}\right]$ and $\left[x_{b}, \xi_{b}\right]$ are indistinguishable in $\Sigma^{(k)}$, there exist two disturbance functions $w_{a}^{(k)}(t)$ and $w_{b}^{(k)}(t)$ such that:

$$
h\left(x\left(t ;\left[x_{a}, \xi_{a}\right] ; \tilde{u} ; w_{a}^{(k)}\right)\right)=h\left(x\left(t ;\left[x_{b}, \xi_{b}\right] ; \tilde{u} ; w_{b}^{(k)}\right)\right)
$$

$\forall t \in\left[0, t_{1}+t_{2}+\cdots+t_{g-1}+t_{g}\right) \subset \mathcal{I}$. On the other hand, by taking the two quantities in (7) at $t=t_{1}+t_{2}+\cdots+t_{g-1}+$ $t_{g}$, we can consider them as functions of the $g$ arguments $t_{1}, t_{2}, \cdots, t_{g}$. Hence, by differentiating with respect to all these variables, we also have:

$$
\begin{aligned}
& \frac{\partial^{g} h\left(x\left(t_{1}+\cdots+t_{g} ;\left[x_{a}, \xi_{a}\right] ; \tilde{u} ; w_{a}^{(k)}\right)\right)}{\partial t_{1} \partial t_{2} \cdots \partial t_{g}}= \\
& =\frac{\partial^{g} h\left(x\left(t_{1}+\cdots+t_{g} ;\left[x_{b}, \xi_{b}\right] ; \tilde{u} ; w_{b}^{(k)}\right)\right)}{\partial t_{1} \partial t_{2} \cdots \partial t_{g}}
\end{aligned}
$$

By computing the previous derivatives at $t_{1}=t_{2}=\cdots=$ $t_{g}=0$ and by using proposition 1 we obtain, if $g \leq k$ :

$$
\mathcal{L}_{\theta_{1} \theta_{2} \cdots \theta_{g}}^{g} h \mid \begin{aligned}
& x_{a}=x_{\theta_{1} \theta_{2} \cdots \theta_{g}}^{g} h \mid \begin{array}{l}
x=x_{b} \\
\xi=\xi_{a}
\end{array} \\
& \xi=\xi_{b}
\end{aligned}
$$

where $\theta_{h}=f_{0}^{(k)}+\sum_{i=1}^{m_{u}} f_{i}^{(k)} u_{i}^{h}, h=1, \cdots, g$. The equality in (9) must hold for all possible choices of $u_{1}^{h}, \cdots, u_{m_{u}}^{h}$. By appropriately selecting these $u_{1}^{h}, \cdots, u_{m_{u}}^{h}$, we finally obtain:

$$
\mathcal{L}_{v_{1} v_{2} \cdots v_{g}}^{g} h\left|\begin{array}{l}
x_{x}=x_{a} \\
\xi=\xi_{a}
\end{array}=\mathcal{L}_{v_{1} v_{2} \cdots v_{g}}^{g} h\right| \begin{aligned}
& x=x_{b} \\
& \xi=\xi_{b}
\end{aligned}
$$

where $v_{1} v_{2} \cdots v_{g}$ are vector fields belonging to the set $\left\{f_{0}^{(k)}, f_{1}^{(k)}, \cdots, f_{m_{u}}^{(k)}\right\}$

In [19] it was also defined the concept of $V$-indistinguishable states, with $V$ a subset of the definition set (in the specific case $V \subseteq M^{(k)}$ ) that includes the two considered states. From this definition and the previous proof we can alleviate the assumptions in the previous proposition. Specifically, we have the following:

Remark 1 The statement of proposition 3 also holds if $\left[x_{a}, \xi_{a}\right]$ and $\left[x_{b}, \xi_{b}\right]$ are $V$-indistinguishable, being $V$ any open subset of $M^{(k)}$ and $\left[x_{a}, \xi_{a}\right],\left[x_{b}, \xi_{b}\right] \in V$.

Thanks to the results stated by propositions 2 and 3 we will introduce the extension of the observability rank condition in the next section.

\section{EXTENSION OF THE OBSERVABILITY RANK CONDITION}

According to the observability rank condition, the weak local observability of the system in (1) with $m_{w}=0$ at a given point $x_{0}$ can be investigated by analyzing the codistribution generated by the gradients of the Lie derivatives of its output. Specifically, if the dimension of this codistribution is equal to the dimension of the state on a given neighbourhood of $x_{0}$, we conclude that the state is weakly locally observable at $x_{0}$ (theorem 3.1 in [19]). We can also check the weak local observability of a subset of the state components. Specifically, a given component of the state is weakly locally observable at $x_{0}$, if its gradient belongs to the aforementioned codistribution ${ }^{1}$. The proof of theorem 3.1 in [19] is based on the fact that all the Lie

\footnotetext{
${ }^{1} \mathrm{~A}$ component of the state is observable at $x_{0}$ if it is constant on the indistinguishable set of $x_{0}\left(I_{x_{0}}\right)$.
} 
derivatives (up to any order) of the output computed along any direction allowed by the system dynamics take the same values at the states which are indistinguishable.

Let us consider now the general case, i.e., when $m_{w} \neq$ 0 . In the extended system $\left(\Sigma^{(k)}\right)$ we know that the Lie derivatives up to the $k$-order satisfy the same property (see proposition 3). Therefore, we can extend the validity of theorem 3.1 in [19] to our case, provided that we suitably augment the state and that we only include the Lie derivatives up to the $k$-order to build the observable codistribution.

In the sequel, we will introduce the following notation:

- $\bar{\Omega}_{m}$ will denote the observable codistribution for $\Sigma^{(k)}$ that includes all the Lie derivatives of the output along $f_{0}^{(k)}, f_{1}^{(k)}, \cdots, f_{m_{u}}^{(k)}$ up to the order $m \leq k$;

- The symbol $d$ will denote the gradient with respect to the extended state in (2);

- For a given codistribution $\Lambda$ and a given vector field $\eta$, we will denote by $\mathcal{L}_{\eta} \Lambda$ the codistribution whose covectors are the Lie derivatives along $\eta$ of the covectors in $\Lambda$ (we are obviously assuming that the dimension of these covectors coincides with the dimension of $\eta$ ).

- Given two vector spaces $V_{1}$ and $V_{2}$, we will denote with $V_{1}+V_{2}$ their sum, i.e., the span of all the generators of both $V_{1}$ and $V_{2}$.

- For a given $V \subseteq M^{(k)}$ and a given $\left[x_{0}, \xi_{0}\right] \in V$, we will denote with $I_{\left[x_{0}, \xi_{0}\right]}^{V}$ the set of all the states $V$-indistinguishable from $\left[x_{0}, \xi_{0}\right]$.

The codistribution $\bar{\Omega}_{m}$ can be computed recursively by the following algorithm:

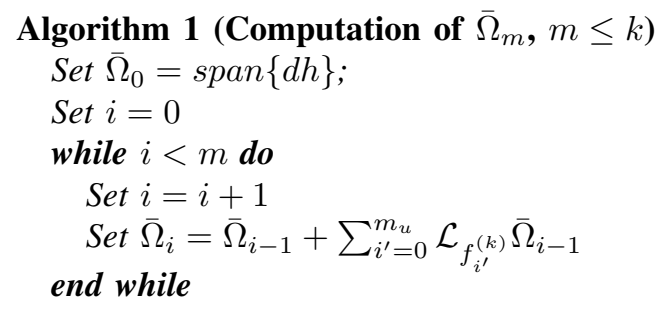

Let us denote by $x_{j}$ the $j^{\text {th }}$ component of the state $(j=$ $1, \cdots, n)$. We introduce the following definition:

Definition $2(E O R C)$ For the system $\Sigma^{(k)}$, the $j^{\text {th }}$ component of the state (i.e., $x_{j}, j=1, \cdots, n$ ) satisfies the extended observability rank condition at $\left[x_{0}, \xi_{0}\right]$ if $d x_{j} \in \bar{\Omega}_{k}$ at $\left[x_{0}, \xi_{0}\right]$. If this holds $\forall j=1, \cdots, n$, we say that the state $x$ satisfies the extended observability rank condition at $\left[x_{0}, \xi_{0}\right]$ in $\Sigma^{(k)}$.

We have the following result, which is the extension of the result stated by theorem 3.1 in [19]:

Proposition 4 For $\Sigma^{(k)}$, if $x_{j}(j=1, \cdots n)$ satisfies the extended observability rank condition at $\left[x_{0}, \xi_{0}\right]$, then $x_{j}$ is weakly locally observable at $\left[x_{0}, \xi_{0}\right]$. Additionally, $x_{j}$ remains weakly locally observable by further extending the state (i.e., in every system $\left.\Sigma^{(f)}(f>k)\right)$.
Proof: We prove that it exists an open neighbourhood $U$ of $\left[x_{0}, \xi_{0}\right]$ such that, for every open neighbourhood $V \subseteq U$ of $\left[x_{0}, \xi_{0}\right], x_{j}$ is constant on the set $I_{\left[x_{0}, \xi_{0}\right]}^{V}$. Since $d x_{j} \in \bar{\Omega}_{k}$ at $\left[x_{0}, \xi_{0}\right]$, it exists some open neighborhood $U$ of $\left[x_{0}, \xi_{0}\right]$, such that $x_{j}$ can be expressed in terms of the Lie derivatives of $h$ along the directions $f_{i^{\prime}}^{(k)}\left(i^{\prime}=0,1, \cdots, m_{u}\right)$ up to the $k$ order. If $V \subseteq U$ is an open neighborhood of $\left[x_{0}, \xi_{0}\right]$, then proposition 3 and remark 1 imply that all the Lie derivatives up to the $k$ order are constant on the set $I_{\left[x_{0}, \xi_{0}\right]}^{V}$ and, consequently, also $x_{j}$ is constant on this set. Finally, the fact that $x_{j}$ is weakly locally observable in every system $\Sigma^{(f)}(f>k)$ directly follows from proposition 2

In accordance with the previous result, the $E O R C$ is a tool to analyze the observability properties of a nonlinear system driven by known and unknown inputs. However, we remark two important limitations of the EORC. The former consists in the fact that the state augmentation can be continued indefinitely. As a result, the EORC only provides sufficient conditions for the weak local observability of the state components. The latter regards the computational cost demanded to check if it is satisfied. Specifically, the computation demanded to check if $d x_{j}$ belongs to $\bar{\Omega}_{k}$ can be very complex because by increasing $k$ we also increase the dimension of the extended state.

\section{ApPlications}

We apply the EORC described in section IV in order to investigate the observability properties of two nonlinear systems driven by unknown inputs. We also consider the case of multiple outputs. The first system characterizes a localization problem in two dimensions in the framework of mobile robotics. For this simple example, the use of the $E O R C$ is not required to derive the observability properties, which can be obtained by using intuitive reasoning.

The second system is much more complex and describes one of the most important sensor fusion problem, which is the problem of fusing visual and inertial measurements. We will refer to this problem as to the visual-inertial structure from motion problem (the Vi-SfM problem). This problem has been investigated by many disciplines, both in the framework of computer science [7], [22], [23], [29], [34] and in the framework of neuroscience (e.g., [5], [10], [11]). Inertial sensors usually consist of three orthogonal accelerometers and three orthogonal gyroscopes. All together, they constitute the Inertial Measurement Unit (IMU). We will refer to the fusion of monocular vision with the measurements from an IMU as to the standard Vi-SfM problem. In [20], [22], [23], [26], [27], [29], [33] and [36] the observability properties of the standard Vi-SfM have been investigated in several different scenarios. Very recently, following two independent procedures, the most general result for the standard $\mathrm{Vi}$ SfM problem has been provided in [16] and [30]. This result can be summarized as follows. In the standard $\mathrm{Vi}^{-}$ SfM problem all the independent observable states are: the positions in the local frame of all the observed features, the three components of the speed in the local frame, the biases 
affecting the inertial measurements, the roll and the pitch angles, the magnitude of the gravity and the transformation between the camera and IMU frames. The fact that the yaw angle is not observable is an obvious consequence of the system invariance under rotation about the gravity vector. We want to use here the theory developed in the previous sections in order to investigate the observability properties of the Vi-SfM problem when the number of inertial sensors is reduced, i.e., when the system is driven by unknown inputs.

\section{A. Simple 2D localization problem}

We consider a vehicle that moves on a $2 D$-environment. The configuration of the vehicle in a global reference frame, can be characterized through the vector $\left[x_{v}, y_{v}, \theta_{v}\right]^{T}$ where $x_{v}$ and $y_{v}$ are the cartesian vehicle coordinates, and $\theta_{v}$ is the vehicle orientation. We assume that the dynamics of this vector satisfy the unicycle differential equations:

$$
\left[\begin{array}{l}
\dot{x}_{v}=v \cos \theta_{v} \\
\dot{y}_{v}=v \sin \theta_{v} \\
\dot{\theta}_{v}=\omega
\end{array}\right.
$$

where $v$ and $\omega$ are the linear and the rotational vehicle speed, respectively, and they are the system inputs. Additionally, we assume that the vehicle is equipped with a GPS able to provide its position. Hence, the system output is the following two-components vector:

$$
y=\left[x_{v}, y_{v}\right]^{T}
$$

Let us start by considering the case when both the system inputs, i.e., the two functions $v(t)$ and $\omega(t)$, are available. By comparing (1) with (11) we obtain $x=\left[x_{v}, y_{v}, \theta_{v}\right]^{T}$, $m_{u}=2, m_{w}=0, u_{1}=v, u_{2}=\omega, f_{0}(x)=\left[\begin{array}{lll}0,0 & 0\end{array}\right]^{T}$, $f_{1}(x)=\left[\cos \theta_{v}, \sin \theta_{v}, 0\right]^{T}$ and $f_{2}(x)=\left[\begin{array}{ll}0,0,1\end{array}\right]^{T}$.

In order to investigate the observability properties, we apply the observability rank crondition introduced in [19].

The system has two outputs: $h_{x} \equiv x_{v}$ and $h_{y} \equiv y_{v}$. By definition, they coincide with their zero-order Lie derivatives. Their gradients with respect to the state are, respectively: $[1,0,0]$ and $[0,1,0]$. Hence, the space spanned by the zero-order Lie derivatives has dimension two. Let us compute the first order Lie derivatives. We obtain: $\mathcal{L}_{1}^{1} h_{x}=\cos \theta_{v}$, $\mathcal{L}_{1}^{1} h_{y}=\sin \theta_{v}, \mathcal{L}_{2}^{1} h_{x}=\mathcal{L}_{2}^{1} h_{y}=0$. Hence, the space spanned by the Lie derivatives up to the first order span the entire configuration space and we conclude that the state is weakly locally observable.

We now consider the case when both the system inputs are unknown. In this case, by comparing (1) with (11) we obtain $m_{u}=0, m_{w}=2, w_{1}=v, w_{2}=\omega, f_{0}(x)=\left[\begin{array}{lll}0,0 & 0\end{array}\right]^{T}$, $g_{1}(x)=\left[\cos \theta_{v}, \sin \theta_{v}, 0\right]^{T}$ and $g_{2}(x)=\left[\begin{array}{ll}0,0,1 & 1\end{array}\right]^{T}$.

Intuitively, we know that the knowledge of both the inputs is unnecessary in order to have the full observability of the entire state. Indeed, the first two state components can be directly obtained from the GPS. By knowing these two components during a given time interval, we also know their time derivatives. In particular, we know $\dot{x}_{v}(0)$ and $\dot{y}_{v}(0)$.
From (11) we easily obtain: $\theta_{v}(0)=\operatorname{atan}\left(\frac{\dot{y}_{v}(0)}{\dot{x}_{v}(0)}\right)$. Hence, also the initial orientation is observable, by only using the GPS measurements.

Let us proceed by applying the $E O R C$, discussed in section IV. We start by computing the codistribution $\bar{\Omega}_{0}$ in $\Sigma^{(0)}$. We easily obtain:

$$
\bar{\Omega}_{0}=\operatorname{span}\{[1,0,0],[0,1,0]\}
$$

From this we know that $x_{v}$ and $y_{v}$ are weakly locally observable. We want to know if also $\theta_{v}$ is weakly locally observable (in which case the entire state would be weakly locally observable). We have to compute $\bar{\Omega}_{1}$ in $\Sigma^{(1)}$. For, we build the system $\Sigma^{(1)}$. We have: ${ }^{1} x=$ $\left[x_{v}, y_{v}, \theta_{v}, v, \omega\right]^{T}$. We can easily obtain the analytical expression for the quantities appearing in (3). We have: $f_{0}^{(1)}(x)=\left[\cos \theta_{v} v, \sin \theta_{v} v, \omega, 0,0\right]^{T}$. We compute the analytical expression of the first-order Lie derivatives along this vector filed. We have: $\mathcal{L}_{0}^{1} h_{x}=\nabla h_{x} \cdot f_{0}^{(1)}(x)=$

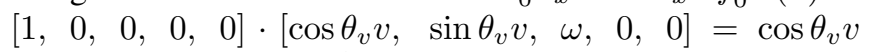
(similarly, we obtain $\mathcal{L}_{0}^{1} h_{y}=\sin \theta_{v} v$ ). We obtain:

$$
\begin{gathered}
\bar{\Omega}_{1}=\operatorname{span}\{[1,0,0,0,0],[0,1,0,0,0], \\
\left.\left[0,0,-\sin \theta_{v} v, \cos \theta_{v}, 0\right],\left[0,0, \cos \theta_{v} v, \sin \theta_{v}, 0\right]\right\}
\end{gathered}
$$

from which we obtain that the gradient of $\theta_{v}$ belongs to $\bar{\Omega}_{1}$. Therefore, also $\theta_{v}$ is weakly locally observable and so the entire original state.

\section{B. The Vi-SfM with partial input knowledge}

For the brevity sake, we do not provide here the computation necessary to deal with this problem. All the details are available in [30] (see also the work in [28] for the definition of continuous symmetries). Here we provide a summary of these results. First of all, we remark that the Vi-SfM problem can be described by a nonlinear system with six inputs (3 are the accelerations along the three axes, provided by the accelerometers, and 3 are the angular speeds provided by the gyroscopes). The outputs are the ones provided by the vision. In the simplest case of a single point feature, they consist of the two bearing angles of this point feature in the camera frame.

We analyzed the following three cases:

1) camera extrinsically calibrated, only one input known (corresponding to the acceleration along a single axis);

2) camera extrinsically uncalibrated, only one input known (corresponding to the acceleration along a single axis);

3) camera extrinsically uncalibrated, two inputs known (corresponding to the acceleration along two orthogonal axes).

The dimension of the original state is 12 in the first case and 23 in the other two cases. Additionally $m_{u}=1$ and $m_{w}=5$ in the first two cases while $m_{u}=2$ and $m_{w}=4$ in the last case.

In [30] we prove that the observability properties of $\mathrm{Vi}$ SfM do not change by removing all the three gyroscopes 
and one of the accelerometers. In other words, exactly the same properties hold when the sensor system only consists of a monocular camera and two accelerometers. To achieve this result, we computed the Lie derivatives up to the second order for the third case mentioned above. By removing a further accelerometer (i.e., by considering the case of a monocular camera and a single accelerometer) the system loses part of its observability properties. In particular, the distribution $\Delta^{k}\left(\equiv \bar{\Omega}_{k}^{\perp}\right), \forall k \geq 2$, contains a single vector. This vector describes a continuous symmetry that is the invariance under the rotation around the accelerometer axis. This means that some of the internal parameters that define the extrinsic camera calibration, are no longer identifiable. Although this symmetry does not affect the observability of the absolute scale and the magnitude of the velocity, it reflects in an indistinguishability of all the initial speeds that differ for a rotation around the accelerometer axis. On the other hand, if the camera is extrinsically calibrated (i.e., if the relative transformation between the camera frame and the accelerometer frame is known (first case mentioned above)) this invariance disappears and the system still maintains full observability, as in the case of three orthogonal accelerometers and gyroscopes. The analysis of this system (the first case mentioned above) has been done in the extreme case when only a single point feature is available. This required to significantly augment the original state. In particular, in [30] we compute all the Lie derivatives up to the $7^{\text {th }}$ order, i.e., we included in the original state the 5 unknown inputs together with their time-derivatives up to the six order. We prove that the gradient of any component of the original state, with the exception of the yaw angle, is orthogonal to the distribution $\Delta^{k}, \forall k \geq 7$ (see the computational details in [30]) ${ }^{2}$.

\section{Conclusion}

In this paper we investigated the problem of nonlinear observability when part (or even all) of the system inputs is unknown. We made the assumption that the unknown inputs are differentiable functions of time (up to a given order). The goal was not to design new observers but to provide simple analytic conditions in order to check the weak local observability of the state. An unknown input was also called disturbance.

We started the analysis by providing a new definition of indistinguishable states. Then, in order to separate the effect of the known inputs from the effect of the unknown inputs on the system outputs, the original state was augmented by including the unknown inputs together with their time derivatives up to a given order. This allowed us to obtain the extension of basic properties, which hold in the standard case (i.e., when all the inputs are known). Starting from these properties, the paper provided an analytic condition which

\footnotetext{
${ }^{2}$ Note that, the yaw angle is not observable even in the case when all the 6 inputs are known. The fact that the yaw is unobservable is a consequence of a symmetry in the considered system, which is the system invariance under rotations about the gravity axis.
}

guarantees the weak observability of the state. This condition was called the extended observability rank condition.

The extended observability rank condition has been illustrated by deriving the observability properties of several nonlinear systems driven by unknown inputs.

We remark two important limitations of the EORC. The former consists in the fact that the state augmentation can be continued indefinitely. As a result, the EORC only provides sufficient conditions for the weak local observability of the state components. The latter regards the computational cost demanded to check if it is satisfied. Specifically, the computation demanded to check if $d x_{j}$ belongs to $\bar{\Omega}_{k}$ can be very complex because by increasing $k$ we also increase the dimension of the extended state.

In [31], [32], we focused our attention on these fundamental issues:

- understanding if there exists a given $\hat{k}$ such that, if $d x_{j} \notin \bar{\Omega}_{\hat{k}}$, then $d x_{j} \notin \bar{\Omega}_{k} \forall k>\hat{k}$.

- obtaining a new codistibution $\left(\Omega_{k}\right)$ that is the span of covectors whose dimension is $n$ (i.e., independent of the state extension) such that $d_{x} x_{j} \in \Omega_{k}$ if and only if $d x_{j} \in \bar{\Omega}_{k}$ (where $d_{x}$ denotes the gradient with respect to the original state $x$ );

In [31], [32] we fully addressed both these issues in the case $m_{w}=1$. Specifically, we proved that the observable codistribution can be splitted into two codistributions. The former is generated by the gradients of scalar functions that only depend on the original state. The latter is generated by the gradients of scalar functions that depend on the entire augmented state. However, this latter codistribution can be ignored when deriving the observability properties of the original state. The former codistribution, namely the one generated by the gradients of scalar functions that only depend on the original state, is defined by a simple recursive algorithm. In [31], [32] we also provided a complete answer to the first issue by proving that this algorithm converges in a finite number of steps and by also providing the criterion to establish that the convergence of the algorithm has been reached (theorem 2 in [32]). Also this proof is based on several tricky analytic steps. Our current investigation is focused on extending the results in [31], [32] to the case of multiple unknown inputs $\left(m_{w}>1\right)$.

\section{REFERENCES}

[1] J.-P. Barbot, M. Fliess, T. Floquet, "An algebraic framework for the design of nonlinear observers with unknown inputs", IEEE Conf. on Decision and Control, New-Orleans, USA, 2007

[2] J.-P. Barbot, D. Boutat, T. Floquet ,"An observation algorithm for nonlinear systems with unknown inputs", Automatica, Volume 45, Issue 8, August 2009, Pages 1970-1974

[3] G. Basile and G. Marro. On the observability of linear, time invariant systems with unknown inputs. J. Optimization Theory Appl., 3:410 415, 1969.

[4] F. A. W. Belo, P. Salaris, and A. Bicchi, 3 Known Landmarks are Enough for Solving Planar Bearing SLAM and Fully Reconstruct Unknown Inputs, The 2010 IEEE/RSJ International Conference on Intelligent Robots and Systems October 18-22, 2010, Taipei, Taiwan

[5] A. Berthoz, B. Pavard and L.R. Young, Perception of Linear Horizontal Self-Motion Induced by Peripheral Vision Basic Characteristics and Visual-Vestibular Interactions, Exp. Brain Res. 23, 471-489 (1975). 
[6] S.P. Bhattacharyya Observer design for linear systems with unknown inputs IEEE Transactions on Automatic Control, 23 (1978)

[7] M. Bryson and S. Sukkarieh, Observability Analysis and Active Control for Airbone SLAM, IEEE Transaction on Aerospace and Electronic Systems, vol. 44, no. 1, 261-280, 2008

[8] Chen, W. and Saif, M. (2006). Unknown input observer design for a class of nonlinear systems: an LMI approach. In IEEE American Control Conference.

[9] Darouach, M., Zasadzinski, M., Xu, S. J. (1994). Full-order observers for linear systems with unknown inputs. IEEE Transactions on Automatic Control, 39(3)

[10] Dokka K., MacNeilage P. R., De Angelis G. C. and Angelaki D. E., Estimating distance during self-motion: a role for visual-vestibular interactions, Journal of Vision (2011) 11(13):2, 1-16

[11] C. R. Fetsch, G. C. DeAngelis and D. E. Angelaki, Visual-vestibular cue integration for heading perception: Applications of optimal cue integration theory, Eur J Neurosci. 2010 May ; 31(10): 1721-1729

[12] Floquet, T., Barbot, J. (2004). A sliding mode approach of unknown input observers for linear systems. In 43rd IEEE conference on decision and control. Atlantis, Paradise Island, Bahamas. December.

[13] Floquet, T., Edwards, C., Spurgeon, S. (2007). On sliding mode observers for systems with unknown inputs. Int. J. Adapt. Control Signal Process Vol 21, no. 89, 638-656, 2007.

[14] Guan, Y., Saif, M. (1991). A novel approach to the design of unknown input observers. IEEE Transactions on Automatic Control, 36(5).

[15] R. Guidorzi and G. Marro. On Wonham stabilizability condition in the synthesis of observers for unknown-input systems. Automatic Control, IEEE Transactions on, 16(5):499-500, oct 1971.

[16] Chao X. Guo and Stergios I. Roumeliotis, IMU-RGBD Camera 3D Pose Estimation and Extrinsic Calibration: Observability Analysis and Consistency Improvement, ICRA 2013, Karlsruhe, Germany

[17] Q.P. Ha, H. Trinh State and input simultaneous estimation for a class of nonlinear systems Automatica, 40 (2004).

[18] H. Hammouri and Z. Tmar. Unknown input observer for state affine systems: A necessary and sufficient condition. Automatica, 46(2):271$278,2010$.

[19] Hermann R. and Krener A.J., 1977, Nonlinear Controllability and Observability, Transaction On Automatic Control, AC-22(5): 728-740

[20] J. A. Hesch, D. G. Kottas, S. L. Bowman, and S. I. Roumeliotis, Towards consistent vision-aided inertial navigation, Int. Workshop on the Algorithmic Foundations of Robotics, Cambridge, MA, Jun, 2012.

[21] M. Hou, P.C. Mller Design of observers for linear systems with unknown inputs IEEE Transactions on Automatic Control, 37 (6) (1992).

[22] E. Jones and S. Soatto, "Visual-inertial navigation, mapping and localization: A scalable real-time causal approach", The International Journal of Robotics Research, vol. 30, no. 4, pp. 407-430, Apr. 2011.

[23] J. Kelly and G. Sukhatme, Visual-inertial simultaneous localization, mapping and sensor-to-sensor self-calibration, Int. Journal of Robotics Research, vol. 30, no. 1, pp. 56-79, 2011.

[24] Koenig, D. and Mammar, S. (2001). Design of a class of reduced order unknown inputs nonlinear observer for fault diagnosis. In IEEE American Control Conference.

[25] D. Koening, B. Marx, D. Jacquet Unknown input observers for switched nonlinear discrete time descriptor system IEEE Transactions on Automatic Control, 53 (1) (2008).

[26] D. G. Kottas, J. A. Hesch, S. L. Bowman, and S. I. Roumeliotis, On the consistency of vision-aided inertial navigation, in Proc. of the Int. Symposium on Experimental Robotics, Canada, Jun 2012.

[27] M. Li and A. I. Mourikis, Improving the accuracy of EKF-based visualinertial odometry, ICRA 2012, St. Paul, MN

[28] A. Martinelli, State Estimation Based on the Concept of Continuous Symmetry and Observability Analysis: the Case of Calibration, IEEE Transactions on Robotics, vol. 27, no. 2, pp. 239-255, 2011

[29] A. Martinelli, Vision and IMU data fusion: closed-form solutions for attitude, speed, absolute scale and bias determination, IEEE Transactions on Robotics, Volume 28 (2012), Issue 1 (February), pp 44-60.

[30] A. Martinelli, Observability Properties and Deterministic Algorithms in Visual-Inertial Structure from Motion, Foundations and Trends in Robotics, 139-209, http://dx.doi.org/10.1561/2300000030, 2014

[31] A. Martinelli, Nonlinear Unknown Input Observability: Analytical expression of the observable codistribution in the case of a single unknown input, Proceedings of the SIAM Conference on Control and Its Applications (CT15), July 2015, Paris, France.
[32] A. Martinelli, Non linear Unknown Input Observability: Extension of the observability rank condition and the case of a single unknown input, INRIA Technical report (available on line on the HAL archive http://hal.archives-ouvertes.fr/)

[33] Mirzaei F.M. and Roumeliotis S.I., A Kalman filter-based algorithm for IMU-camera calibration: Observability analysis and performance evaluation, Transactions on Robotics, 2008, Vol. 24, No. 5, 1143-1156

[34] D. Strelow and S. Singh, Motion estimation from image and inertial measurements, International Journal of Robotics Research, 23(12), 2004

[35] S.H. Wang, E.J. Davison, P. Dorato Observing the states of systems with unmeasurable disturbance IEEE Transactions on Automatic Control, 20 (1975)

[36] Weiss., S., Vision Based Navigation for Micro Helicopters, PhD thesis, Diss. ETH No. 20305

[37] F. Yang, R.W. Wilde Observer for linear systems with unknown inputs IEEE Transactions on Automatic Control, 33 (7) (1988). 